\title{
A multicenter randomized controlled trial to assess the feasibility of testing modified ultrafiltration as a blood conservation technology in cardiac surgery
}

\author{
Munir Boodhwani, MD, MMSc, FRCSC, ${ }^{a}$ Andrew Hamilton, MD, FRCSC, ${ }^{b}$ Benoit de Varennes, MD, FRCSC, \\ Thierry Mesana, MD, PhD, FRCSC, ${ }^{\mathrm{a}} \mathrm{K}$. Williams, ${ }^{\mathrm{d}}$ George A. Wells, PhD, ${ }^{\mathrm{d}} \mathrm{H}$. Nathan, MD, FRCPC, \\ Jean Yves Dupuis, MD, FRCPC, ${ }^{\mathrm{e}}$ A. Babaev, MD, ${ }^{\mathrm{f}}$ P. Wells, MD, MSc, FRCPC, ${ }^{\mathrm{g}}$ and \\ Fraser D. Rubens, MD, MSc, FRCSC ${ }^{\mathrm{a}}$
}

\begin{abstract}
Objectives: Modified ultrafiltration is a technique after cardiopulmonary bypass whereby blood withdrawn from the aortic cannula is passed across a semipermeable membrane to hemoconcentrate. Unblinded trials have suggested that modified ultrafiltration is efficacious for blood conservation. The objective of this trial was to assess the feasibility of a model testing modified ultrafiltration in which all members of the surgical team were blinded to the intervention.
\end{abstract}

\begin{abstract}
Methods: Patients ( $<65 \mathrm{~kg}$ ) undergoing procedures involving cardiopulmonary bypass were randomized to undergo either modified ultrafiltration $(n=29)$ or sham (circulation without an interposed filter, $n=36)$ for 15 minutes. The circuit was shielded from all members of the team except the perfusionist. A questionnaire was administered to determine the blinding success.
\end{abstract}

\begin{abstract}
Results: Modified ultrafiltration resulted in a removal of $1000 \pm 251 \mathrm{~mL}$ of fluid and a reduction in the pump balance $(1025 \pm 807$ vs $1804 \pm 838 ; P<.001)$ with an increase in hemoglobin immediately after intervention (increase of $7.7 \pm 8.8 \mathrm{~g} / \mathrm{L}$ in modified ultrafiltration vs $3.8 \pm 5.1 \mathrm{~g} / \mathrm{L}$ in sham; $P=.04$ ). Introduction or increase in dose of vasopressors was more frequent in the modified ultrafiltration group $(52 \%$ vs $28 \% ; P=.048)$. Differences in red cell transfusion rates between groups did not reach statistical significance $(P=.59)$. Blinding was successful for the anesthetist (blinding index 0.13 [95\% confidence interval, $0.11-0.38$ ] and the intensivist (blinding index, 0.09 [95\% confidence interval, 0.14-0.31]) but not for the surgeon (blinding index, 0.24 [95\% confidence interval, 0.05-0.42]). The compliance rate for the transfusion protocol was greater than $90 \%$.
\end{abstract}

Conclusions: Modified ultrafiltration was effective for hemoconcentration after cardiopulmonary bypass in patients of low body weight, but it is associated with an increased need for vasopressor support. The anesthetist and intensivist were successfully blinded to the intervention. (J Thorac Cardiovasc Surg 2010;139:701-6)

Cardiac surgery is one of the leading consumers of blood products and it will likely remain so for the foreseeable future. Intraoperative and postoperative blood losses are predictable contributors to this problem. A less commonly recognized factor is the effect of hemodilution of the patient by the pump prime of cardiopulmonary bypass (CPB). This

\footnotetext{
From the Division of Cardiac Surgery, ${ }^{\mathrm{a}}$ University of Ottawa Heart Institute, Ottawa,

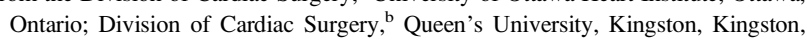
Ontario; Division of Cardiac Surgery, ${ }^{\mathrm{c}}$ Royal Victoria Hospital, Montreal, Quebec; Department of Epidemiology and Community Medicine, ${ }^{\mathrm{d}}$ University of Ottawa, Ottawa, Ontario; Division of Cardiac Anaesthesia, ${ }^{\mathrm{e}}$ University of Ottawa Heart Institute, Ottowa, Ontario; Division of Perfusion, ${ }^{\mathrm{f}}$ University of Ottawa Heart Institute, Ottawa, Ontario; and Department of Medicine, ${ }^{\mathrm{g}}$ University of Ottawa, Ottowa, Ontario, Canada.

Disclosures: None.

This study was funded by grant NA 5565 from the Heart and Stroke Foundation of Ontario.

Received for publication July 13, 2009; revisions received Nov 10, 2009; accepted for publication Nov 23, 2009.

Address for reprints: Fraser D. Rubens, MD, MSc, FRCSC, Professor of Surgery, University of Ottawa Heart Institute, 40 Ruskin St, Ottawa, Ontario K1Y 4W7, Canada (E-mail: frubens@ottawaheart.ca).

0022-5223/\$36.00

Copyright $(c) 2010$ by The American Association for Thoracic Surgery

doi:10.1016/j.jtcvs.2009.11.056
}

hemodilution is especially pronounced in patients of low body weight owing to their lower circulatory volume.

Several strategies have been advocated to minimize the impact of hemodilution, including retrograde autologous priming, forced diuresis, and ultrafiltration. This latter technology involves passing the blood across a semipermeable membrane and using a pressure gradient to remove excessive fluid. The resulting hemoconcentration may improve coagulation owing to the higher hematocrit ${ }^{1-3}$ and by the concentration of coagulation factors. ${ }^{1,2}$ Low molecular weight components, which may include cytokines and toxins, are also removed owing to the membrane pore size, thus potentially decreasing perioperative inflammation. ${ }^{4,5}$

Conventional ultrafiltration involves the interposition of the filter device in series within the CPB circuit. However, solute removal can only be achieved while the patient is still supported by $\mathrm{CPB}$; thus, to remove more fluid, $\mathrm{CPB}$ times must be prolonged. On the other hand, with modified ultrafiltration (MUF), fluid removal occurs after coming off $\mathrm{CPB}$. The cannulas are left in the ascending aorta and the right atrium. Fluid is withdrawn from the aortic line and 


\section{Abbreviations and Acronyms \\ $\mathrm{BI}=$ blinding index \\ $\mathrm{CPB}=$ cardiopulmonary bypass \\ MUF $=$ modified ultrafiltration}

passed through the filter device, with the hemoconcentrated product returned through a side port on the venous cannula. When the intervention is completed, the cannulas are removed and heparin is reversed.

Pediatric cardiac surgeons have long espoused the benefits of this technology. Several groups have also studied this approach in adult cardiac surgery, and their data suggest that this technology may be successful as a blood conservation strategy. ${ }^{3,6,7}$ There are several limitations of the previous trials that may explain why MUF has not been widely used despite these positive findings. First, none of these trials was blinded and thus bias may have affected transfusion and other treatment decisions. Second, inasmuch as this procedure is somewhat inconvenient, the magnitude of the benefit demonstrated may not have been sufficient to influence current practice. The trials may have been compromised in their design by the failure to target this technology in patients who are both at high risk for transfusion and most likely to benefit from hemoconcentration after $\mathrm{CPB}$, such as those patients with low body weight. Therefore, a definitive trial that would influence practice would include blinding of the intervention and targeting the strategy to the highest risk population. Before this trial was undertaken, a pilot trial was completed to (1) determine the success of intraoperative blinding of the intervention, (2) determine the compliance to the intervention (ie, how often randomized patients can proceed to the intervention and compliance with transfusion protocols), (3) determine issues in screening, recruitment, and safety, and (4) collect data to inform sample size calculation and hypothesis generation.

We hypothesized that whereas the intensivist should be easily blinded, blinding of the surgeon may be more difficult and the anesthesiologist even more so. If all are successfully blinded ( $>85 \%$ success), in a definitive trial we would propose to use the current planned intervention. If we are unable to blind the anesthesiologist, decisions for transfusion would be made by the surgeon. If neither the surgeon nor the anesthesiologist can be successfully blinded, this will be important information that would render this aspect of the model "unblindable"; thus, in the definitive trial, there would be no reason to use a sham circuit.

\section{METHODS}

We conducted a prospective, multicenter, randomized controlled trial in the Ottawa Heart Institute, the Kingston General Hospital (1 surgeon), and the Royal Victoria Hospital, Montreal (1 surgeon) between August 2006 and January 2009. The study protocol was approved by the human research ethics board at each center and written informed consent was obtained from all patients. Eligible patients included those with weight of $65 \mathrm{~kg}$ or less or body surface area less than $1.7 \mathrm{~m}^{2}$. Patients were excluded for the following reasons: emergency surgery, off-pump surgery, abciximab use within 7 days of surgery, inability to obtain consent, age less than 18 years, surgeon refusal, preoperative hemoglobin level less than $100 \mathrm{~g} / \mathrm{dL}$, preoperative hemodialysis, and refusal to accept blood products.

A narcotic-based anesthetic was used. Tranexamic acid (a 1-g bolus after induction and a $2.0-\mathrm{mg} \cdot \mathrm{kg}^{-1} \cdot \mathrm{h}^{-1}$ intraoperative infusion) was administered to all study patients. Antiplatelet and antithrombotic medication was stopped before surgery according to best practice. After sternotomy, patients were given heparin to achieve an activated clotting time greater than 400 seconds. CPB was conducted with a roller pump, a membrane oxygenator (COBE CML Duo; COBE Cardiovascular Inc, Arvada, Colo), a 43- $\mu \mathrm{m}$ arterial filter (COBE Sentry with PrimeGard), a closed venous reservoir bag, ascending aortic cannula, and 2-stage or double venous cannula return. All of the pumps were primed with $1300 \mathrm{~mL}$ of Ringer lactate. Bypass flows were maintained at 2.4 to $3.2 \mathrm{~L} \cdot \mathrm{m}^{-2} \cdot \mathrm{min}^{-1}$. The heart was arrested with antegrade cold blood cardioplegic solution. During cardiac anoxia, the body temperature was reduced to a systemic temperature of $34^{\circ} \mathrm{C}$, and at the completion of the procedure patients were rewarmed, with care taken to never exceed $37^{\circ} \mathrm{C}$ (nasopharyngeal).

Two-way stopcocks were attached to the arterial and venous cannulas. Treatment was randomly assigned and the intervention was commenced once the patient was off $\mathrm{CPB}$ in a stable state and the surgeon had verified control of surgical bleeding such that protamine would normally be administered. The venous line was then drained, as is routine, to empty all blood into the reservoir in both groups. The inflow tubing for the intervention circuit was then passed up to the operating site with the inlet attached to the stopcock on the arterial line. After the tubing had been de-aired, the outlet was attached to a stopcock on the venous cannula in the right atrium and a clamp was placed distally such that all reinfusion occurred into the right atrium. In the test (MUF) group $(\mathrm{n}=29)$, suction $(-150 \mathrm{~mm} \mathrm{Hg})$ was applied to an interposed hemofilter (UF 70; Terumo Inc, Tokyo, Japan). This hemofilter was chosen because it provides the most rapid rate of filtration among current commercially available devices. ${ }^{8}$ In the control group (sham; $n=36$ ), the circuit was identical with the exception that there was no interposed filter and sham flow was kept at $200 \mathrm{~mL} / \mathrm{min}$. During the intervention, the perfusionist maintained a stable mean blood pressure with a target range within $10 \%$ of starting mean blood pressure by slow readministration of the pump reservoir volume through the arterial line. When the pump reservoir was empty, the cardiotomy reservoir (above the pump reservoir) could no longer be used for sucker blood owing to standard technical design. Thus, further mediastinal drainage was transferred to a blood collection device (eg, Atrium device; Atrium Medical Corporation, Hudson, NH). Autotransfusion (after protamine) was the same for control and experimental groups according to each institution's practice. The intervention was continued for 15 minutes in both groups. In the test group, if it was determined by the perfusionist that no further volume could be removed before the target time, suction was discontinued and circuit flow was maintained at $200 \mathrm{~mL} / \mathrm{min}$. In both groups, at the end of the intervention, the venous cannulas were removed, protamine was administered, and residual pump volume was infused slowly as per standard practice.

\section{Objectives of the Study}

The objectives of the study were as follows:

1. To determine the success of blinding

2. To determine the compliance rate of the clinical model

3. To refine the ascertainment of outcomes and obtain estimates of event rates.

\section{Study Outcomes}

We proposed a priori that the most important outcome derived from this pilot project was related to the success of intraoperative blinding. Blinding was measured using a blinding index (BI) as described by Bang, $\mathrm{Ni}$, and 
Davis. ${ }^{9}$ The BI proposed is scaled to an interval of -1 to 1 , with 1 being complete lack of blinding, 0 being consistent with perfect blinding, and -1 indicating opposite guessing, which may be related to unblinding. If a 1-sided $95 \%$ confidence limit of the BI does not include 0 , the treatment arm is deemed to fail to achieve blinding.

The BI was ascertained on the basis of a questionnaire administered to the anesthesiologist and surgeon after each operation and to the intensivist after 24 hours in the intensive care unit. The questionnaire offered 5 levels of response, based on their perception of the treatment allocation to which they think the patient was assigned:

Code 1: "Strongly believe the treatment was MUF."

Code 2: "Somewhat believe the treatment was MUF."

Code 3: "Somewhat believe the treatment was sham."

Code 4: "Strongly believe the treatment was sham."

Code 5: "Don't know."

Individuals who decline an opinion ("don't know") were subsequently asked to choose a treatment allocation anyway for a separate analysis. Compliance was measured by the frequency with which randomized patients proceed to the intervention as well as the compliance with the postintervention transfusion protocol.

\section{Sample size}

The sample size calculation was based on the primary outcome regarding the success of blinding. To determine the sample size for the present trial, we calculated the SD of the BI from an example given by Bang, Ni, and Davis ${ }^{9}$ based on data from a cholesterol lowering trial.

\section{Randomization-Allocation Concealment}

Randomization was done in blocks, with sizes ranging from 4 to 8 and with the block size unknown to the investigators. The randomization process consisted of a computer-generated random listing of treatment allocations, stratified by center. Treatment was assigned by a telephone call made to the central office in Ottawa just before the operation to enable the perfusionist to prepare the shielded sham or MUF circuits.

\section{Blinding (Masking)}

All members of the surgical team except the perfusionist (who did not make transfusion decisions) were blinded to treatment allocation. A shield was placed to obscure the pertinent part of the ultrafiltration or "sham" circuit from the surgeon and the anesthesiologist. Flow was maintained through the "sham" circuit at $200 \mathrm{~mL} / \mathrm{min}$ during the intervention so that the visible circuits appear the same. The return tubing was also wrapped in paper tape to blind the surgeon with regard to the degree of oxygenation of the blood (during MUF, oxygen is extracted from the returning blood; thus it is darker than the sham circuit).

The perfusionist recorded the hemoglobin level at the end of the intervention and informed the anesthesiologist and the surgeon as to whether it was above or below $70 \mathrm{~g} / \mathrm{L}$ only. If necessary, the anesthesiologist could request to be unblinded to the actual value if oxygen delivery was still inadequate (eg, presence of a base deficit $>5 \mathrm{mmol} / \mathrm{L}$ or a cardiac index $<2 \mathrm{~L} \cdot \mathrm{min}^{-1}$. $\mathrm{m}^{-2}$ ) such that hemoglobin levels needed to be manipulated or in case of active bleeding that could not be managed in a blinded fashion. Transfusions were given in the intensive care unit with the same threshold; however, the intensivist (although blinded to treatment group) would not be blinded to the actual hemoglobin or hematocrit result. The study coordinator in all cases recorded the indication for the transfusion after consultation with the attending physician.

\section{Statistical Methods}

Continuous data are presented as mean \pm standard deviation for normally distributed data and median (interquartile range) for nonnormally distributed data. Continuous variables were compared by a 2 -tailed Student $t$ test for normally distributed data and Wilcoxon rank sum test for nonnormally distributed data. Categorical variables were compared by the $\chi^{2}$ test or Fisher's exact test when the cell count in any one cell was less than 5 . Statistical analyses were performed with SAS version 9.1 (SAS Institute Inc, Cary, NC). The primary end point of blinding index was calculated as previously described by Bang, $\mathrm{Ni}$, and Davis. ${ }^{9}$

\section{RESULTS}

\section{Patient Population and Study Intervention}

Patient flow during screening, recruitment, and randomization is depicted in Figure 1. Demographics of the patients randomized (Table 1) and hematologic, hemodynamic, and perfusion data (Table 2) were similar. Analysis was by intent-to-treat. The intraoperative intervention was successfully implemented in all patients. MUF was used to remove an average of $1000 \pm 21 \mathrm{~mL}$ of fluid in the treated group, resulting in a significant difference in the net fluid administered at the end of CPB (corrected pump balance) between groups $(1804 \pm 838$ vs $1025 \pm 807 \mathrm{~mL}$, sham vs MUF; $P<.001)$. MUF also resulted in a greater increase in hematocrit $(0.011 \pm 0.015$ vs $0.024 \pm 0.026$, sham vs MUF; $P=.03)$ and hemoglobin levels $(3.8 \pm 5.1 \mathrm{~g} / \mathrm{L}$ vs $7.7 \pm 8.8 \mathrm{~g} / \mathrm{L}$, sham vs MUF; $P=.04)$. No adverse events were associated with either intervention.

\section{Clinical Outcomes}

There was no significant difference in the units of red blood cells transfusioned after intervention between groups $(2.6 \pm 4.3$ vs $2.0 \pm 1.73$ units/patient, sham vs MUF; $P=.59$ ). There was no difference in the transfusion rates when patients who had surgical bleeding were removed from the calculation (data not shown). The proportion of patients transfused after the intervention was $74 \%$ in the entire cohort $(67 \%$ sham, $83 \%$ MUF; $P=.14)$. Overall blood product use, including packed red cells, plasma, and platelets, was also similar between groups $(6.8 \pm 11.6$ vs $5.5 \pm 7.3$ units/patient, sham vs MUF; $P=.6$ ). Postoperatively, there was no significant difference in hemoglobin levels between groups $(91 \pm 12$ vs. $85 \pm 14 \mathrm{~g} / \mathrm{L}$, sham vs MUF; $P=.12$ ). Compliance with the transfusion protocol was $91 \%$ by the anesthesiologists intraoperatively, $90 \%$ in the intensive care unit, and $100 \%$ thereafter until patient discharge. Five patients in the study required reopening owing to bleeding or tamponade $(\mathrm{MUF}=3, \mathrm{SHAM}=2$ ). There was no difference in chest tube output between the 2 groups $(712 \pm 517$ vs $579 \pm 332 \mathrm{~mL} / 12$ hours, sham vs MUF; $P=.21)$.

Fifteen minutes after intervention, cardiac index was lower in the MUF group than in the sham group $(1.9 \pm 0.4$ vs $\left.2.3 \pm 0.5 \mathrm{~L} \cdot \min ^{-1} \cdot \mathrm{m}^{-2} ; P=.004\right)$. Furthermore, a larger proportion of MUF patients had vasopressors started or increased in dosage in the intensive care unit $(52 \%$ vs $28 \%$; $P=.048$ ). The total duration of vasopressor use also tended to be higher in the MUF patients $(12.6 \pm 25.5$ vs 


\section{Consort Flowchart - TRAM Trial}

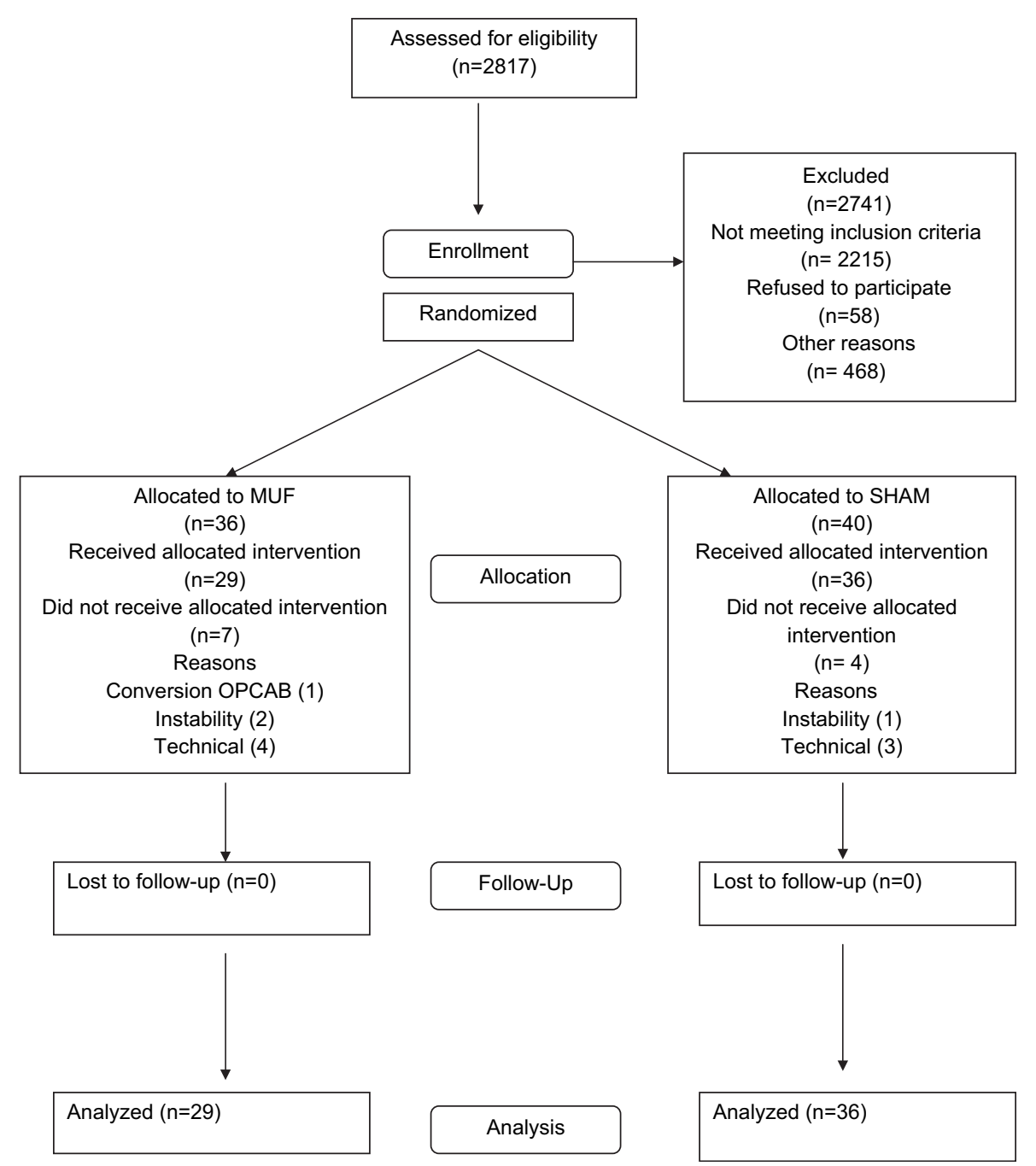

FIGURE 1. Consolidated Standards of Reporting Trials (CONSORT) diagram of patient flow. MUF, Modified ultrafiltration group; SHAM, control group; $O P C A B$, off-pump coronary artery bypass grafting; TRAM, transfusion reduction and modified ultrafiltration.

$5.2 \pm 11.1$ hours; $P=.054$ ). There was no in-hospital mortality, and length of hospital stay was similar between groups $(9.6 \pm 9.8$ vs $8.9 \pm 5.6$ days, sham vs MUF; $P=.71)$.

\section{Assessment of Blinding}

The BI for the surgeon in the MUF group was 0.034 (95\% confidence interval $[\mathrm{CI}],-0.199$ to $0.268 ; P>$ $.05)$. In the sham group, the BI for the surgeon was 0.229 (95\% CI, 0.052 to $0.406 ; P<.05)$. The BI for the anesthesiologist in the MUF group was $0.138(95 \%$ $\mathrm{CI},-0.112$ to $0.388 ; P>.05)$. The $\mathrm{BI}$ in the sham group was $0.028(95 \% \mathrm{CI},-0.191$ to $0.247 ; P>.05)$. The BI for the intensivist was $0.091(95 \% \mathrm{CI},-0.143$ to 0.325 ; $P>.05)$ in the MUF group and 0.083 (95\% CI, -0.132 to $0.298 ; P>.05)$ in the sham group.

\section{CONCLUSIONS}

In this pilot trial assessing the feasibility of a randomized double-blind controlled trial comparing MUF and sham circuits in cardiac surgery, the efficacy of blinding of the surgeon, the anesthesiologist, and the intensivist was successfully tested. For the surgeon, there was insufficient evidence to show unblinding in the MUF group, but there was a significant excess of correct guesses in the sham group, such that one can determine that the surgeon could not be blinded. For the anesthesiologist, there was an excess of correct guesses in the MUF group, but this was not statistically significant $(P>.05)$, and in the sham group, there was insufficient evidence to confirm unblinding $(P<.05)$. There was no significant evidence to confirm unblinding in the intensivist. Finally, there was excellent $(>90 \%)$ compliance 
TABLE 1. Patient demographics

\begin{tabular}{lccc}
\hline & MUF & Sham & $\boldsymbol{P}$ value \\
\hline Age (y) & $66.3 \pm 13.1$ & $71.1 \pm 10.1$ & .11 \\
Sex (\% female) & 65.5 & 77.8 & .27 \\
BSA & $2.21 \pm 2.08$ & $2.47 \pm 4.19$ & .06 \\
ASA (\%) & 83.7 & 61.1 & .06 \\
Clopidogrel (\%) & 27.6 & 11.1 & .09 \\
Heparin & 6.9 & 8.3 & .83 \\
Low molecular weight heparin (\%) & 6.9 & 2.8 & .43 \\
CCS class III or IV (n) & 41 & 25 & .16 \\
NYHA class III or IV (n) & 21 & 25 & .68 \\
Abnormal LV function ( $\geq$ class II) & 34 & 17 & .1 \\
Left main (\%) & 14 & 17 & .75 \\
Diabetes (\%) & 24 & 8.3 & .08 \\
Hypertension (\%) & 55 & 55 & .98 \\
History of MI (\%) & 21 & 25 & .68 \\
Hypercholesterolemia (\%) & 45 & 44 & .98 \\
Smoker (\%) & 38 & 25 & .26 \\
\hline
\end{tabular}

$M U F$, Modified ultrafiltration; $B S A$, body surface area; $A S A$, acetylsalicylic acid use within 7 days of surgery; $C C S$, Canadian Cardiovascular Society (angina class); $N Y H A$, New York Heart Association (congestive heart failure class); $L V$, left ventricular; $M I$, myocardial infarction

with regard to the transfusion protocol by the anesthesiologist after the intervention as well as by the intensivist in the first 24 hours after surgery.

As expected, when patients underwent MUF, they experienced a significant increase in their hematocrit value, which was associated with a significant decrease in their corrected pump balance. The group that underwent the intervention, however, was more likely to require vasopressor support or to have their inotropes increased within the first 24 hours after the operation. This may be related to uncompensated hypovolemia, and it would be valuable to measure this in further studies. There was no statistically significant difference in the exposure to transfusion products between the groups undergoing MUF or Treatment Response Assessment Method, but this trial was not powered to detect this difference as a primary outcome.

MUF has been proposed as an attractive blood conservation technology. ${ }^{7}$ It is a relatively inexpensive means to remove excessive crystalloid in patients undergoing CPB, and we hypothesized that its potential positive effects would be more easily demonstrated in small patients who are at highest risk of hemodilution. In the total population studied in this trial, more than $80 \%$ of patients are exposed to blood products. It is also of note that this population has a high proportional representation of women, and thus studies of this sort have a positive bias to address this pressing concern in this group who are normally excluded from surgical research. ${ }^{10}$

There are several key findings that have implications for a definitive trial:

1. We randomized before induction to allow the perfusionist to set up the circuit in advance so as not to "waste"
TABLE 2. Demographics; baseline hematology, and bypass indices

\begin{tabular}{lccc}
\hline & MUF & Sham & $P$ value \\
\hline Preoperative hemoglobin $(\mathrm{g} / \mathrm{dL})$ & $129 \pm 17$ & $136 \pm 11$ & .04 \\
Preoperative hematocrit $(\%)$ & $0.38 \pm 0.05$ & $0.40 \pm 0.03$ & .12 \\
Preintervention hemoglobin $(\mathrm{g} / \mathrm{dL})$ & $84 \pm 13$ & $86 \pm 11$ & .39 \\
Platelets $\left(10^{3} / \mathrm{mm}^{3}\right)$ & $263 \pm 90$ & $256 \pm 69$ & .73 \\
International normalized ratio & $1.0 \pm 0.1$ & $1.2 \pm 0.6$ & .48 \\
aPTT $(\mathrm{s})$ & $29.5 \pm 7.8$ & $32.4 \pm 21.9$ & .47 \\
Creatinine $(\mu \mathrm{mol} / \mathrm{L})$ & $83 \pm 22$ & $82 \pm 20$ & .97 \\
CI at induction $\left(\mathrm{L} \cdot \mathrm{min}^{-1} \cdot \mathrm{m}^{-2}\right)$ & $1.96 \pm 0.37$ & $2.16 \pm 0.71$ & .46 \\
Anesthesia volume $(\mathrm{mL})$ & $1750 \pm 1005$ & $1599 \pm 834$ & .55 \\
Pump time (mm) & $88 \pm 29$ & $95 \pm 33$ & .38 \\
Crossclamp time (mm) & $65 \pm 23$ & $67 \pm 21$ & .69 \\
Lowest hematocrit on CPB $(\%)$ & $0.24 \pm 0.04$ & $0.24 \pm 0.03$ & .77 \\
Pump balance (mL) & $2025 \pm 884$ & $1805 \pm 838$ & .31 \\
Inotropes to come off CPB $(\%)$ & 28 & 17 & .29 \\
\hline MUF, Modified ultrafiltration; $a P T T$, activated partial thromboplastin time; $C I$, cardiac \\
index; $C P B$, cardiopulmonary bypass. & \multicolumn{3}{l}{}
\end{tabular}

ultrafiltration devices. Unexpectedly, we had a high percentage of cases excluded by the surgeon after randomization but before induction, unlike the trial of Luciani and associates. ${ }^{7}$ Therefore, resources should be available to allow randomization at the point of protamine administration, preparing each patient as part of the MUF group with splicing out of the filter if the randomization is to control.

2. Inasmuch as the surgeon could not be blinded successfully, it is unnecessary to bind the tubing with tape. The surgeon could still be kept blinded to hematocrit results and instructed not to participate in transfusion decisions.

3. The intervention was successful (ie, $50 \%$ of the pump balance removed in 15 minutes) in $59 \%$ of patients. The target of this removal threshold was chosen on the basis of the most common practice in the literature ${ }^{6}$; on the basis of the current study, perhaps an additional 5 minutes should be considered to augment this.

4. This study was difficult to complete and expensive owing to the complexity of the model and the need for careful supervision with regard to compliance and blinding. It would be greatly facilitated by educational videos to participating teams demonstrating precise techniques of MUF application and setup.

As expected, there was no statistically significant difference in transfusion rate in the 2 groups because the trial was not powered for this. On the basis of the standard deviations for the units of red cells administered, we can predict that in a definitive trial, to test a hypothesis whereby this technology decreased the exposure of patients to units of red blood cells in the intervention group by $20 \%(0.6$ units/patient [2.0 vs 2.6 ; SD, 1.7 units]), one would need 182 patients per group (alpha, .05; power, 80\%).

The major limitation of this trial, as described above, related to the impact of inadvertent withdrawal of patients 
after randomization and before intervention. This could be effectively addressed by changing the protocol in the definitive trial. It is emphasized that this was a pilot trial specifically designed to identify these methodologic issues.

In summary, in a randomized controlled trial assessing the feasibility of comparing MUF with sham in CPB, the anesthesiologists and the intensivists were successfully blinded to the intervention and there was a high compliance rate with the transfusion protocol. MUF successfully effected hemoconcentration by decreasing the corrected pump prime, but it is not clear whether this results in a decrease in the subsequent exposure to blood products. It appeared that patients undergoing MUF were more likely to require vasopressor support after the intervention. It would be feasible, although difficult, to address the question of the efficacy of MUF in a larger randomized controlled trial. Such a trial would have to include important safety measures regarding excessive hypovolemia that may place the patients at risk to lower postoperative cardiac output with the intervention, and this information would be essential before considering universal application of this technology.

We recognize the assistance of our research coordinators: Sarika Naidoo, Suzanne Picard, Marlie Poirier, and Rebecca Tee (Ottawa), Carole Albert (Montreal), and Debbie Shore (Kingston). We acknowledge the contribution of the perfusionists, surgeons, intensiv- ists, and anesthesiologists who participated in the trial with their patients.

\section{References}

1. Ootaki Y, Yamaguchi M, Oshima Y, Yoshimura N, Oka S. Effects of modified ultrafiltration on coagulation factors in pediatric cardiac surgery. Surg Today. 2002;32:203-6

2. Friesen RH, Campbell DN, Clarke DR, Tornabene MA. Modified ultrafiltration attenuates dilutional coagulopathy in pediatric open heart operations. Ann Thorac Surg. 1997;64:1787-9.

3. Leyh RG, Bartels C, Joubert-Hubner E, Bechtel JF, Sievers HH. Influence of modified ultrafiltration on coagulation, fibrinolysis and blood loss in adult cardiac surgery. Eur J Cardiothorac Surg. 2001;19:145-51.

4. Chew MS, Brix-Christensen V, Ravn HB, Brandslund I, Ditlevsen E, Pedersen J, et al. Effect of modified ultrafiltration on the inflammatory response in paediatric open-heart surgery: a prospective, randomized study. Perfusion. 2002;17:327-33.

5. Tassani P, Richter JA, Eising GP, Barankay A, Braun SL, Haehnel CH, et al. Influence of combined zero-balanced and modified ultrafiltration on the systemic inflammatory response during coronary artery bypass grafting. J Cardiothorac Vasc Anesth. 1999;13:285-91.

6. Boodhwani M, Williams K, Babaev A, Gill G, Saleem N, Rubens FD. Ultrafiltration reduces blood transfusions following cardiac surgery: a meta-analysis. Eur $J$ Cardiothorac Surg. 2006;30:892-7.

7. Luciani GB, Menon T, Vecchi B, Auriemma S, Mazzucco A. Modified ultrafiltration reduces morbidity after adult cardiac operations: a prospective, randomized clinical trial. Circulation. 2001;104(12 Suppl. 1):I253-9.

8. Boldt J, Zickmann B, Fedderson B, Herold C, Dapper F, Hempelmann G. Six different hemofiltration devices for blood conservation in cardiac surgery. Ann Thorac Surg. 1991;51:747-53.

9. Bang H, Ni L, Davis CE. Assessment of blinding in clinical trials. Control Clin Trials. 2004;25:143-56.

10. Shevde K, Pagala M, Kashikar A, Tyagaraj C, Shahbaz N, Iqbal M, et al. Gender is an essential determinant of blood transfusion in patients undergoing coronary artery bypass graft procedure. J Clin Anesth. 2000;12:109-16. 